\title{
Lactobacillus Fermented Extract
}

National Cancer Institute

\section{Source}

National Cancer Institute. Lactobacillus Fermented Extract. NCI Thesaurus. Code C74053.

A proprietary dietary supplement. Lactobacillus fermented extract is made from soymilk fermented by several strains of lactobacillus and yeast via a symbiotic co-culturing technology. The composition of the agent includes amino acids, vitamins, minerals, fatty acids, isoflavones, and saponins. Lactobacillus fermented extract may support healthy intestinal function. 